\title{
Evidence from In Vivo Imaging That Synaptogenesis Guides the Growth and Branching of Axonal Arbors by Two Distinct Mechanisms
}

\author{
Martin P. Meyer and Stephen J Smith \\ Department of Molecular and Cellular Physiology, Beckman Center, Stanford University, Stanford, California 94305
}

To explore the relationship between axon arbor growth and synaptogenesis, developing retinal ganglion cell (RGC) axon arbors in zebrafish optic tectum were imaged in vivo at high temporal and spatial resolution using two-photon microscopy. Individual RGC axons were dually labeled by expression of a cytosolic red fluorescent protein (DsRed Express) to mark arbor structure and a fusion of the synaptic vesicle protein synaptophysin with green fluorescent protein (Syp:GFP) to mark presynaptic vesicles. Analysis of time-lapse sequences acquired at $10 \mathrm{~min}$ intervals revealed unexpectedly rapid kinetics of both axon branch and vesicle cluster turnover. Nascent axonal branches exhibited short average lifetimes of $19 \mathrm{~min}$, and only $17 \%$ of newly extended axonal processes persisted for periods exceeding $3 \mathrm{~h}$. The majority (70\%) of Syp:GFP puncta formed on newly extended axonal processes. Syp:GFP puncta also exhibited short average lifetimes of $30 \mathrm{~min}$, and only $34 \%$ of puncta were stabilized for periods exceeding $3 \mathrm{~h}$. Moreover, strongly correlated dynamics of Syp:GFP puncta and branch structure suggest that synaptogenesis exerts strong influences on both the extension and the selective stabilization of nascent branches. First, new branches form almost exclusively at newly formed Syp:GFP puncta. Second, stabilized nascent branches invariably bear Syp:GFP puncta, and the detailed dynamics of branch retraction suggest strongly that nascent synapses can act at branch tips to arrest retraction. These observations thus provide evidence that synaptogenesis guides axon arbor growth by first promoting initial branch extension and second by selective branch stabilization.

Key words: axon; imaging; synapse; GFP; retinal ganglion cell; retinotectal; zebrafish

\section{Introduction}

Studies of development of the visual system of nonmammalian vertebrates have contributed greatly to our current understanding of the mechanisms that establish neural circuitry. In fish and frogs, growing retinal ganglion cell (RGC) axons navigate from the retina to their midbrain target, the optic tectum, where they elaborate an axonal arbor and establish synaptic connections with tectal cell dendrites. By guiding retinal axons, molecular cues are believed to convey a topographic map of the visual field present in the retina into the tectum. The basic topographic order is then fine-tuned through dynamic rearrangement of axonal and dendritic arbors, during which branches are continually added and lost, and shifts in this dynamic equilibrium can lead to growth or pruning of the arbor (O'Rourke et al., 1994; Ruthazer et al., 2003; Niell et al., 2004).

Factors that have been shown to shape axonal arbor growth include not only extracellular guidance cues (Yates et al., 2001; Hindges et al., 2002) but also synaptic activity (O'Rourke et al.,

\footnotetext{
Received Aug. 26, 2005; revised March 1, 2006; accepted March 2, 2006.

This work was supported by the National Institutes of Health and the Vincent Coates Foundation. We thank C Garner, C. Larsen, J. Jontes, and D. Lyons for their comments on this manuscript, Sarah Emerson for help with statistical analysis, and C. Niell for MATLAB programming. GAL4-VP16/UAS and Brn3C constructs were kindly provided by $S$. Fraser and H. Baier, respectively.

Correspondence should be addressed to Martin P. Meyer at the above address. E-mail: martinm@stanford.edu. D0I:10.1523/JNEUROSCI.0223-06.2006

Copyright $\odot 2006$ Society for Neuroscience $\quad$ 0270-6474/06/263604-11\$15.00/0
}

1994; Debski and Cline, 2002; Ruthazer and Cline, 2004) and intracellular and intercellular signaling (Hall et al., 2000; CohenCory and Lom, 2004; Govek et al., 2005). Similarly, progress has been made in elucidating the molecular mechanisms by which synapses are assembled (Goda and Davis, 2003; Li and Sheng, 2003; Ziv and Garner, 2004). There have been very few reports, however, of studies designed to explore the detailed relationship between arbor growth and synaptogenesis. The few studies that have addressed the relationship between axon arbor growth and synaptogenesis suggest that these processes may be mechanistically coupled. The idea that synapse formation can direct both dendritic and axonal arborization was proposed over a decade ago as the "synaptotropic hypothesis" of arbor growth, based on electron microscopic analysis of fixed tissue (Vaughn and Sims, 1978; Vaughn, 1989). Synapses could potentially direct growth of neuronal arbors by stimulating formation of new branches, by stabilizing nascent branches, or both. We have recently demonstrated that, by selectively stabilizing filopodia, synaptogenesis guides growth of zebrafish tectal cell dendrites in vivo (Niell et al., 2004). Dynamic imaging of Xenopus RGCs and motor neuron axonal arbors suggests that synapse formation promotes new branch formation (Alsina et al., 2001; Javaherian and Cline, 2005). These studies suggest that synapses guide growth of tectal dendritic, and retinal axonal arbors by selective stabilization of branches in the case of dendrites and by promoting branch formation in the case of axons. However, the possibility that synapse 
formation can also selectively stabilize nascent axonal branches has not been investigated.

To address this question directly we have performed longterm, high temporal resolution, in vivo imaging of RGC axon arbor growth and synaptogenesis in live zebrafish larvae.

\section{Materials and Methods}

Generation of Syp:GFP and Syp:GFP-DSR. Searches of the GenBank database identified a zebrafish expressed sequence tag clone encoding a protein with high sequence homology to mammalian Synaptophysin. The partial coding sequence was used to design PCR primers, and 3' rapid amplification of cDNA ends was performed using the GeneRacer kit (Invitrogen, Carlsbad, CA) to obtain the 3' sequence of zebrafish Synaptophysin from cDNA prepared from adult zebrafish brain. Fulllength Synaptophysin was PCR amplified with primers that generated a $5^{\prime}$ EcoRI site and Kozak consensus sequence immediately $5^{\prime}$ to the translational start codon, and a triglycine linker and a SmaI site at the $3^{\prime}$ terminus of Synaptophysin. The PCR-amplified Synaptophysin was subcloned into pEGFP-N2 (Clontech, Mountain View, CA) to generate an in-frame fusion to the $\mathrm{N}$ terminus of EGFP (enhanced green fluorescent protein). An upstream activator sequence (UAS) and an E1b basal promoter (Koster and Fraser, 2001) was subcloned as a single fragment into the HindIII and EcoRI sites upstream of Synaptophysin to generate Syp: GFP. The UAS-E1b fragment was also cloned into the HindIII-EcoRI sites of pDsRed-Express-1 (Clontech) to generate UAS-DSR. To generate Syp:GFP-DSR, the region coding for the Synaptophysin:GFP fusion was excised with EcoRI-NotI and subcloned into the same sites in pCASPER (gift from Dr. Camilla Larsen, University of California, Los Angeles, Los Angeles, CA). This placed the Synaptophysin:GFP fusion under the control of five UAS repeats and the Hsp70 basal promoter. The entire Synaptophysin:GFP expression cassette, including the simian virus 40 polyadenylation signal, was excised with HindIII and subcloned in a head-tohead configuration into the HindIII site of UAS-DSR to generate Syp: GFP-DSR. Expression of Syp:GFP and Syp:GFP-DSR was driven using the GAL4-UAS system (Koster and Fraser, 2001). A $5 \mathrm{~kb}$ fragment of the RGC-specific Brn3C promoter (gift from Dr. Herwig Baier, University of California, San Francisco, San Francisco, CA) was used to drive expression of the transcriptional activator GAL4 on a separate plasmid (Brn3CGAL4). Brn3C-GAL4 and either Syp:GFP or Syp:GFP-DSR were coinjected into embryos at the one to two cell stage, resulting in mosaic expression in RGCs. We found that $\sim 20 \%$ of injected embryos showed transgene expression, and typically only one RGC per retina was labeled. Curiously, we found that, in embryos injected with Syp:GFP-DSR, only $\sim 30 \%$ of transgene-expressing cells exhibited both Syp:GFP and DsRed Express fluorescence despite the fact that they are encoded on the same plasmid. This may reflect a degree of instability in these constructs. Only cells expressing both Syp:GFP and DsRed Express were used to correlate arbor growth and synaptogenesis.

Validation of Synaptophysin:GFP as a synaptic marker, and wholemount in situ hybridization. Whole-mount in situ hybridization and vibratome sectioning were performed as described previously (Meyer et al., 2005). Zebrafish were fixed and whole-mount labeled with a monoclonal antibody to SV2 (Hybridoma Bank, Iowa City, IA) as described previously (Westerfield, 1995). Acetylcholine receptors (AChRs) at the neuromuscular junction were labeled by mircoinjection of Alexa 594-tagged $\alpha$-bungarotoxin (Invitrogen) into the yolk sack of $2 \mathrm{~d}$ postfertilization (dpf) zebrafish larvae. To determine the degree of colocalization of Syp: GFP clusters with SV2, and AChR clusters, we scored all Syp:GFP clusters in maximum intensity projections. Syp:GFP clusters found to overlap (indicated by a yellow signal in merged red and green images) with red SV2 and ACh labeling were scored as colocalized. Syp:GFP clusters that remained green in merged images were scored as non-colocalized (see also supplemental material, available at www.jneurosci.org).

DNA preparation and microinjection of zebrafish embryos. Raising, maintaining, and spawning of adult zebrafish were performed as described (Westerfield, 1995). All procedures were approved by the Institutional Animal Care and Use Committee of Stanford University. To generate transgene expression in spinal neurons, the pan-neuronal gold- fish $\alpha-1$ tubulin promoter was used to drive expression of Gal4-VP16 on an activator plasmid (Koster and Fraser, 2001). Preparation of DNA, microinjection of embryos, and raising of embryos were performed as described previously (Niell et al., 2004).

Imaging was performed as described (Niell et al., 2004). Briefly, imaging was performed using a $63 \times / 0.9$ numerical aperture water-immersion objective (Zeiss, Oberkochen, Germany), on a custom-made twophoton laser-scanning microscope, tuned to $920 \mathrm{~nm}$ for simultaneous excitation of green fluorescent protein (GFP) and DsRed Express, and $850 \mathrm{~nm}$ for simultaneous excitation of GFP and Alexa 594-tagged Bungarotoxin.

Image analysis was performed as described previously (Niell et al., 2004), with the following modifications. Analysis of individual optical sections of RGC axons expressing Syp:GFP revealed that there was virtually no overlap of Syp:GFP clusters in the $z$-axis. Thus, because of the planar nature of RGC axon arbors, we were able to perform analyses on maximum intensity projections of the entire imaging volume. Lifetimes of all axonal processes were measured by marking all such processes that appeared during a time-lapse episode. These were scored for total lifetime, as well as number of puncta on each branch in each frame using Open View custom analysis software (N. Ziv, Rappaport Institute, Haifa, Israel). These data were used to generate the average number of puncta on the process over its lifetime. Total arbor lengths were measured using ImageJ software, and extension and retraction events, and punctum intensity and size were measured from high temporal resolution movies using Open View custom analysis software. Puncta were identified as discrete local accumulations in Syp:GFP of more than three native pixels $(0.5 \mu \mathrm{m})$ in diameter. The value for the total length of axonal arbor occupied by Syp:GFP puncta was obtained by multiplying the average punctum size $(0.85 \mu \mathrm{m})$ by the average number of puncta on a $4 \mathrm{dpf}$ RGC cell (50), and expressing this as a percentage of the average total arbor length at $4 \mathrm{dpf}(180 \mu \mathrm{m})$. Statistical analyses were performed using the repeated-measures general linear model function of SPSS 11.5.

The GenBank accession number for zebrafish Synaptophysin is DQ011271.

\section{Results}

\section{Axonal arbor growth and Syp:GFP puncta formation are concurrent}

To visualize synapse formation on growing RGC arbors, we cloned zebrafish Synaptophysin and fused it in-frame to the coding region of GFP to generate Synaptophysin:GFP (Syp:GFP). To image axonal arbor growth and synaptogenesis simultaneously, we constructed a plasmid that coexpressed DsRed Express, a soluble space-filling marker, with Syp:GFP. We refer to this plasmid as Syp:GFP-DSR. Synaptophysin is a synaptic vesicle protein involved in synaptic vesicle exocytosis and endocytosis (Valtorta et al., 2004) and is expressed throughout the developing zebrafish nervous system (supplemental Fig. 1A-G, available at www. jneurosci.org as supplemental material). Synaptophysin fused to GFP has been used extensively as a synaptic vesicle marker in vitro (Nakata et al., 1998; Kaether et al., 2000; Li and Murthy, 2001; Bamji et al., 2003; Pennuto et al., 2003) and in Xenopus motor neurons in vivo (Javaherian and Cline, 2005) (for additional validation, see supplemental Fig. 2, available at www.jneurosci.org as supplemental material). In vitro observations have demonstrated that synaptophysin and other presynaptic components are transported along axons as "transport packets" (Nakata et al., 1998; Ahmari et al., 2000; Kaether et al., 2000; Zhai et al., 2001; Shapira et al., 2003). Our time-lapse imaging of RGCs, described below, also reveals mobile clusters of Syp:GFP (data not shown). However, $61 \pm 1.8$ and $67 \pm 1.7 \%$ of the Syp:GFP clusters that we observe in RGC axons between 3 and $4 \mathrm{dpf}$, and 9 and $10 \mathrm{dpf}$, respectively, are present in the same position in two or more consecutive frames of our time-lapse movies, making it unlikely that these are clusters of Syp:GFP undergoing axonal transport. 
A.
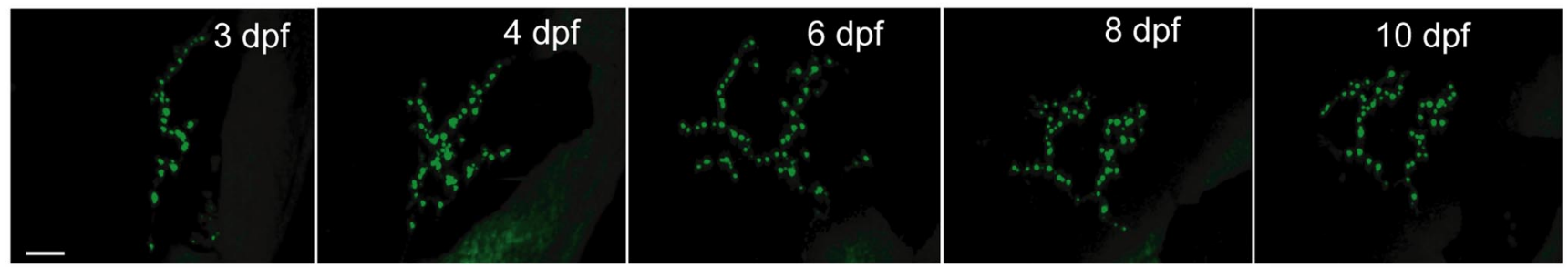

B.

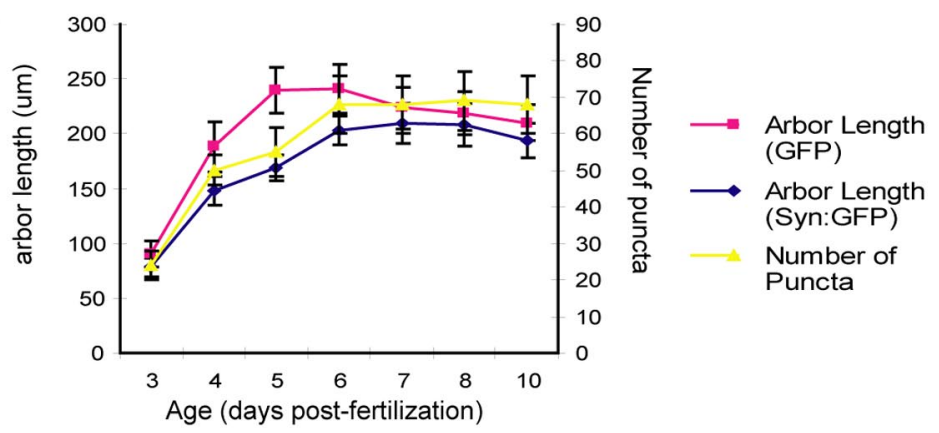

C.

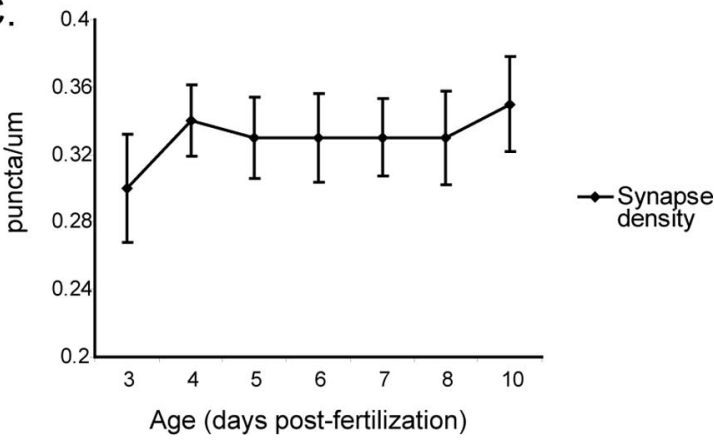

Figure 1. Imaging of RGCs in optic tectum on consecutive days shows that arbor growth and Syp:GFP punctum formation occur simultaneously. $A$, Time series showing the same RGC imaged at $3,4,6,8$, and $10 \mathrm{dpf}$ (indicated in top right corner of each panel). Autofluorescence of the skin is visible in the bottom right corner. Scale bar, $10 \mu \mathrm{m}$. B, Quantification of total branch length and number of puncta. Mean values from eight Syp:GFP-expressing cells (from 8 animals) and seven GFP-expressing cells (from 7 animals) are shown. Error bars indicate SEM. C, Syp:GFP punctum density remains constant during development, indicating that axon arbor growth and synaptogenesis are concurrent.

Together, these data suggest that stable accumulations of Syp: GFP mark presynaptic sites in zebrafish.

In zebrafish, the first RGC axons reach the optic tectum at $\sim 2$ dpf (Stuermer, 1988; Burrill and Easter, 1994), and visually evoked responses can first be elicited $\sim 3 \mathrm{dpf}$ (Easter and Nicola, 1996; Niell and Smith, 2005). Figure $1 A$ shows expression of Syp:GFP in a single RGC that was imaged on consecutive days from 3 to $10 \mathrm{dpf}$ as it arborized in the tectum. A punctate distribution of Syp:GFP, characteristic of presynaptic specializations, is visible even in immature RGC arbors. The number of Syp:GFP puncta increases rapidly between 3 and $6 \mathrm{dpf}$, after which the number of puncta remains relatively stable (Fig. $1 B$ ). RGC axons form synapses with dendrites of type XIV tectal neurons. The time course of Syp:GFP punctum formation in RGCs at $3 \mathrm{dpf}$ correlate quite precisely with the onset of visually evoked responses of zebrafish tectal neurons measured in vivo, providing additional support for the inference that Syp:GFP accumulation represents the formation of synapses (Niell et al., 2005).

Analysis of total arbor length measured from cells expressing Syp:GFP or GFP reveals that arbor length also increases over the same period as Syp:GFP puncta formation (Fig. $1 B$ ). A repeatedmeasures ANOVA shows that total axon branch length increased significantly across the measurement time points $(F=36.47$; lower-bound adjusted $p=0.001$ ). (Tests for within-subject contrasts were significant for linear and quadratic trends over time). Syp:GFP punctum density on axonal branches remains nearly constant (from $0.3 \pm 0.03$ puncta $/ \mu \mathrm{m}$ at $3 \mathrm{dpf}$ to $0.35 \pm 0.03$ puncta $/ \mu \mathrm{m}$ at $10 \mathrm{dpf}$ ) (Fig. $1 C$ ), indicating that there is very little lag time between the extension of a new axon branch and formation of a Syp:GFP punctum on it. The fact that axonal arbor lengths measured from GFP- or Syp:GFP-expressing cells are similar over the 3-10 dpf imaging period suggests that expression of Syp:GFP does not perturb RGC development. (Because of the punctate distribution of Syp:GFP, the finest axonal processes, which were visible in RGCs expressing GFP, could not be visualized in RGCs expressing Syp:GFP. This is likely to account for the lower values of arbor length measured from Syp:GFP cells.) The developmental time course of RGC arbor growth and synapse formation is very similar to that of dendritic arbor growth and synapse formation in tectal neurons in vivo (Niell et al., 2004). This indicates that dendritic and axonal arbor elaboration, and development of presynaptic and postsynaptic specializations in the retinotectal system are concurrent and may be closely coordinated.

\section{Arbor growth and Syp:GFP puncta formation are highly dynamic}

To examine the detailed dynamics of arbor growth and synaptogenesis, we performed high temporal resolution time-lapse imaging of RGC arbors expressing Syp:GFP or Syp:GFP-DSR. Figure $2 A$ shows an image series from a time-lapse session initiated at $3 \mathrm{dpf}$. Analysis of such time-lapse sequences revealed unexpectedly rapid kinetics of nascent axon branch turnover. Timelapse imaging also reveals populations of both stable and transient Syp:GFP puncta (supplemental video 1, available at www.jneurosci.org as supplemental material). Imaging over similar durations between 9 and $10 \mathrm{dpf}$ shows that, in contrast to the situation between 3 and $4 \mathrm{dpf}$, most axon branches and Syp:GFP puncta are stable over many hours (see Fig. $5 C$ ). To compare punctum stability in immature (3-4 dpf) and mature (9-10 dpf) RGC arbors, Syp:GFP-expressing cells were imaged at $20 \mathrm{~min}$ intervals for a period of $15 \mathrm{~h}$. At both developmental stages, there is a population of synapses with short lifetimes as well as a population of synapses with longer lifetimes. Three hours was selected as a threshold to classify synapses into categories of stable or transient. The morphological, functional, and molecular diversity of developing synapses makes a definitive classification of synapse maturity extremely difficult (Ahmari and Smith, 2002). However, our criterion for stability is based on in vitro studies showing that assembly of functional synapses occurs within $1-2 \mathrm{~h}$ of axo-dendritic contact and proceeds at a rapid pace (Ahmari et al., 2000; Friedman et al., 2000; Antonova et al., 2001; Okabe et 
A.
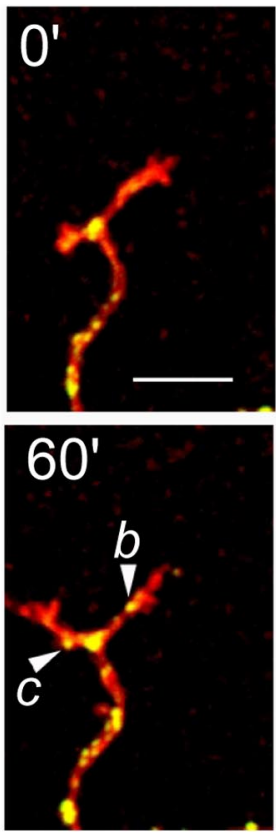
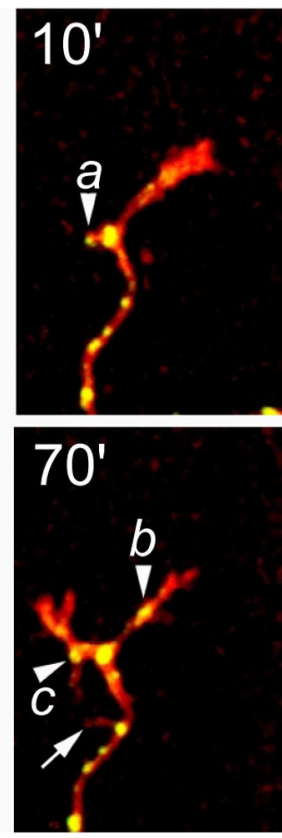
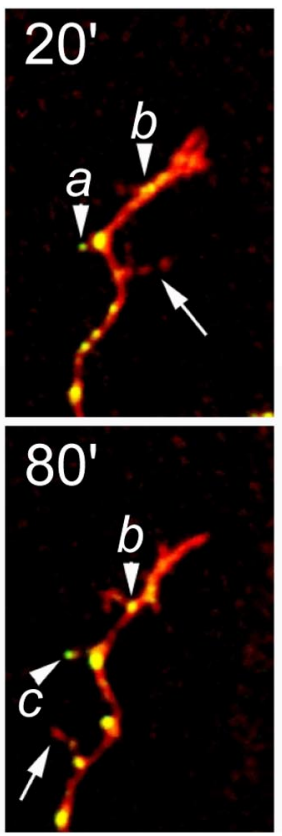
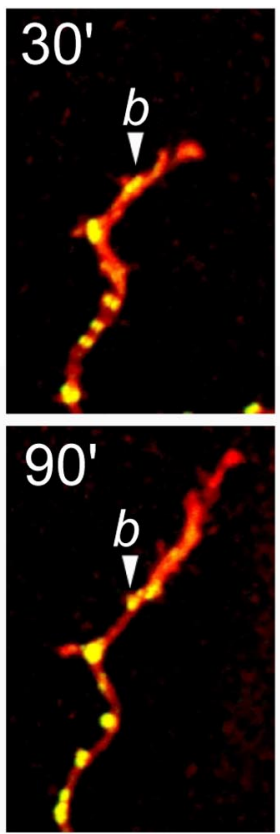
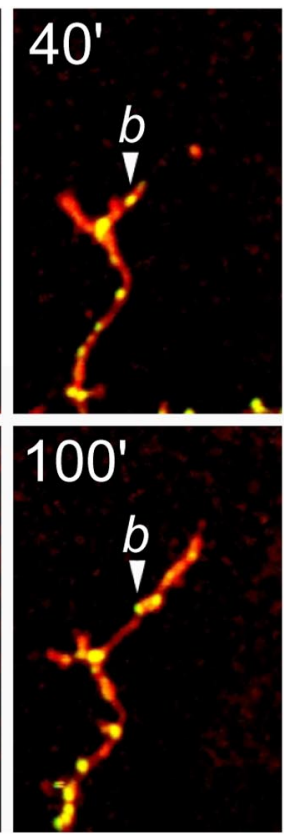
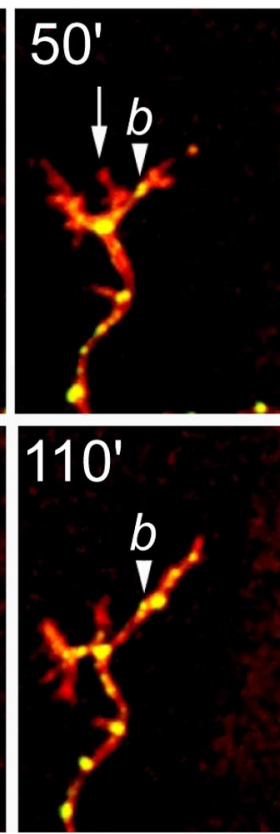

B.

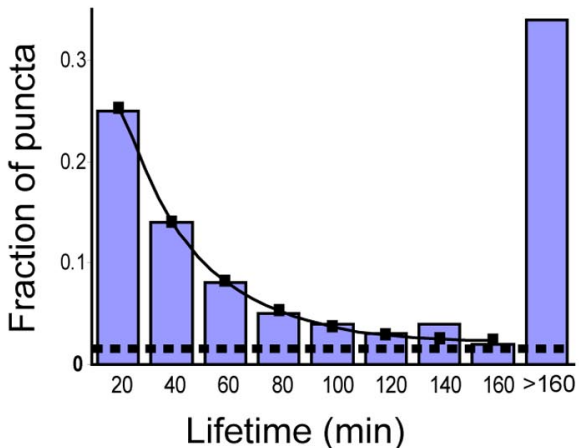

C.

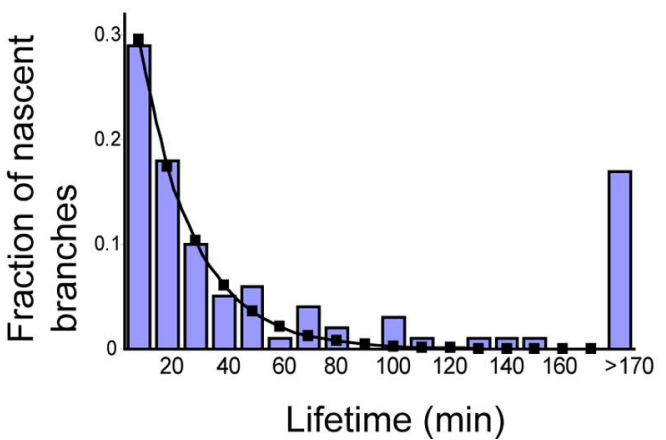

Figure 2. Time-lapse analysis of RGC axon arbor and Syp:GFP punctum dynamics. $A$, Series of stills taken from a time-lapse movie of a 3 dpf RGC expressing Syp:GFP-DSR showing that period of growth is characterized by the rapid formation and elimination of axonal branches and Syp:GFP puncta. Scale bar, $10 \mu \mathrm{m}$. Time in minutes is indicated in the top left corner of each panel. The cell was imaged at $10 \mathrm{~min}$ intervals for a total of $10 \mathrm{~h}$. The arrowheads and arrows indicate examples of new puncta and new nascent branch formation events, respectively. Puncta indicated by the arrowheads labeled $a$ and $c$ were present in only two and three frames, respectively, whereas the puncta indicated by the arrowhead labeled $b$ was present for at least 10 frames. $\boldsymbol{B}$, Lifetime histogram for a total of 605 Syp:GFP puncta (from 5 Syp:GFP-expressing cells imaged in 5 animals at 20 min intervals) observed to form during time-lapse imaging of 3- 4 dpf larvae. The single exponential curve fit to this histogram represents an average punctum lifetime of 30 min and describes closely the lifetime of approximately one-half of punctum lifetimes scored. The dashed asymptote and the large fraction of lifetimes $>3 \mathrm{~h}$ describe subpopulations of puncta with longer average lifetimes. C, Lifetime histogram for a total of 103 nascent axonal branches (from 4 Syp:GFP-DSR-expressing cells imaged in 4 animals at 10 min intervals) observed to form during time-lapse imaging. The single exponential curve fit to this histogram represents an average nascent branch lifetime of $19 \mathrm{~min}$ and describes closely the lifetime distribution of the great majority of the branch lifetimes scored. Note that only $17 \%$ of newly extended nascent branches persisted for $>3 \mathrm{~h}$.

al., 2001). Thus, sites marked by a cluster of Syp:GFP that persist for $>3 \mathrm{~h}$ are likely to possess many of the features that define functional synapses. The $3 \mathrm{~h}$ threshold was also used previously to classify postsynaptic density protein 95 (PSD-95):GFP puncta on tectal cell dendrites as stable or unstable (Niell et al., 2004). Between 3 and $4 \mathrm{dpf}$, only $40 \pm 5 \%$ of puncta persist for $>3 \mathrm{~h}(n=$ 793 puncta from five Syp:GFP-expressing cells), whereas, between 9 and $10 \mathrm{dpf}, 74 \pm 1.7 \%$ of puncta are stable $(n=393$ puncta from four Syp:GFP-expressing cells). Thus, as the arbor matures, the number and fraction of stable Syp:GFP puncta increase markedly. A small fraction of unstable puncta is still apparent at 9-10 dpf, however, indicating that a small degree of synapse turnover persists in more mature RGCs.

To explore the dynamics of putative synapse stabilization on immature RGC axons in more detail, we plot the distribution of new Syp:GFP puncta lifetimes observed by time-lapse imaging of
3-4 dpf larvae as a histogram in Figure $2 B$. This histogram shows that new Syp:GFP puncta exhibit a distribution of lifetimes suggesting multiple punctum subpopulations. Approximately onehalf of the 605 observed Syp:GFP punctum lifetimes are well fit by a single exponential describing an average lifetime of $30 \mathrm{~min}$. The nonzero asymptote used to generate the exponential fit suggests, however, that there is at least one subpopulation of much more long-lived puncta. Puncta that undergo elimination are indistinguishable in their initial appearance from puncta that persist and go on to form long-lasting stable puncta, suggesting that these transient puncta may represent nascent synapses that are eliminated. The idea that Syp:GFP punctum elimination represents synapse turnover is further supported by the observation that PSD-95:GFP punctum lifetimes in tectal cell dendrites correlate well with our measurements of Syp:GFP lifetimes in RGC axons (Niell et al., 2004). The close parallels in turnover of presynaptic 
and postsynaptic assemblies we observe at nascent retinotectal synapses suggest that synapse elimination is a prominent feature of retinotectal development.

Figure $2 C$ is a histogram representing the distribution of lifetimes of 103 new nascent branches, as observed from four Syp:GFP-DSR-expressing cells between 3 and $4 \mathrm{dpf}$. The majority of the new nascent branch lifetimes are well described by a single exponential representing an average lifetime of $19 \mathrm{~min}$. Again, however, the histogram suggests the existence of more than one subpopulation. We observed that $17 \%$ of the observed terminal branches persisted for $3 \mathrm{~h}$ or longer, whereas a single exponential distribution with a $19 \mathrm{~min}$ average lifetime would predict only a small fraction of $1 \%$ would persist this long. The histograms of Figure 2, $B$ and $C$, are thus consistent with populations of both Syp: GFP puncta and nascent branches that are inherently unstable, with average lifetimes of 19 and $30 \mathrm{~min}$, respectively, that are nonetheless subject to stabilization processes that may greatly extend individual punctum or branch lifetimes.

These results confirm those of others showing that the period of rapid arbor growth is characterized by the concurrent elimination and formation of axonal branches (O’Rourke et al., 1994; Alsina et al., 2001; Ruthazer et al., 2003). However, by imaging with far greater temporal resolution (10 min compared with intervals of $1 \mathrm{~h}$ or longer in the studies above), we demonstrate extremely rapid kinetics of nascent branch turnover. Furthermore, we show that the dynamic rearrangement of axonal arbors is accompanied by the formation of many Syp:GFP puncta, only a small subset of which persist, thus leading to a gradual accumulation of stable puncta over time.

\section{Syp:GFP puncta form rapidly in the wake of axonal growth cones and on axonal filopodia}

To perform systematic quantitative analysis of the dynamics of synapse formation on growing RGC axonal arbors, we generated "punctum-centric" movies centered on each individual stable punctum that was observed to appear in time-lapse movies of RGCs expressing Syp:GFP-DSR. Cells were imaged at $10 \mathrm{~min}$ intervals for periods of $10-15 \mathrm{~h}$, starting at $3 \mathrm{dpf}$. Analysis of such movies revealed three modes of stable punctum formation. First, the largest fraction of stable puncta ( $45 \pm 5 \% ; n=17$ from four cells) formed within $20 \mathrm{~min}$ (average number of frames within which synapse appeared, $1.6 \pm 0.2$ ) after the passage of migrating axonal growth cones (Fig. $3 A$; supplemental video 2, available at www.jneurosci.org as supplemental material). Neuronal processes were classified as growth cones if at any time during the observation period their tips displayed a broad splayed morphology and demonstrated exploratory behavior that consisted of extension, retraction, and turning behaviors. A similar mechanism of en passant synapse formation has been described in the un- branched axons of zebrafish spinal neurons (Jontes et al., 2000, 2004) and in hippocampal neurons in vitro (Ahmari et al., 2000).

Figure $3 A$ also shows an example of a new branch forming by splitting of an axonal growth cone. One of the growth cones displays a prolonged period of extension, leaving multiple puncta on the newly formed axon branch. The second growth cone stops extending after formation of a single punctum and is eventually eliminated. We have observed six such bifurcation events in timelapse movies, and in each instance the branch point is marked by a newly formed punctum.

The second mode of stable punctum formation that we observe occurs de novo on shafts of stable axon branches (30 $\pm 7 \%$; $n=12$ puncta from four cells) (data not shown). De novo punctum formation may result from trapping of a mobile transport packet triggered by contact initiated by a postsynaptic cell, as has been demonstrated in hippocampal neurons in vitro (Ahmari et al., 2000), or by splitting of a preexisting punctum.

Finally, of the 38 stable punctum formation events that we analyzed, $25 \pm 6 \%$ ( $n=9$ stable puncta from four cells) occurred on dynamic finer processes that extended and retracted rapidly from existing puncta. Because these fine processes did not display the splayed morphology and turning behavior of growth cones, 
we classified these processes as filopodia. Figure $3 B$ (and supplemental video 3, available at www.jneurosci.org as supplemental material) shows a typical formation event. A filopodium extends from a preexisting punctum located on an axon branch, a punctum forms midway along its length, and the filopodium retracts back to the position of the nascent Syp:GFP punctum, which gradually grows in intensity, and persists on what becomes a new axon branch. Figure $3 \mathrm{C}$ demonstrates multiple puncta appearing on a newly extended filopodium. This example also shows new filopodia sprouting from the newly stabilized punctum, leading to additional growth of the nascent branch. In vivo imaging has demonstrated that iterative rounds of filopodial extension and synapse formation define a fundamental mode of new dendritic branch formation in zebrafish tectal cells (Niell et al., 2004), but this is the first direct observation of this mode of axon arbor growth in vivo.

These results suggest that axonal filopodia and growth cones are involved in establishing synaptic contacts, and also growth and branching of axonal arbors.

\section{Nascent branches arise from newly formed Syp:GFP puncta}

Time-lapse analysis of all new growth cone and filopodium formation events from cells expressing Syp:GFP-DSR $(n=103$ events from four cells) revealed that $92 \%$ of such axonal processes originate from sites of punctum formation. We estimate that Syp:GFP puncta occupy only $\sim 24 \%$ of the total length of the axonal arbor at $4 \mathrm{dpf}$ (see Materials and Methods), indicating an overwhelming bias toward formation of axonal processes at sites marked by a cluster of Syp:GFP. Furthermore, cellular motility across a growing axonal arbor is not uniform. Time-lapse imaging reveals that some parts of the arbor display highly dynamic filopodia and growth cones, whereas other regions are stable and bear no dynamic processes (supplemental video 1, available at www.jneurosci.org as supplemental material). This implies that developmental regulation of axon motility is regulated in a highly localized manner. To test the idea that such a developmental transition could occur at the level of individual synapses, we counted the number of filopodia and growth cones (hereafter referred to collectively as nascent branches) emerging from stable Syp:GFP puncta that were observed from the time of their first appearance and could be imaged for prolonged periods after their initial formation. We found that, of the 38 stable punctum formation events observed, 26 were sites of exploratory behavior by dynamic axonal processes. (Of these, 25 were from puncta that formed in the wake of a growth cone or on a filopodium, suggesting that synapses that form midbranch tend not to promote exploratory behavior.) Of the 38 stable punctum formation events we observed, 16 could be imaged for prolonged periods after the initial formation event (average imaging time, $7.2 \pm 0.6 \mathrm{~h}$ ). Figure 4 shows the total number of nascent branches produced from these 16 puncta per hour of the imaging period. These results clearly show that nascent branches extend preferentially from sites marked by newly formed Syp:GFP puncta and that the level of exploratory behavior from these sites declines with age. Our observation that new nascent branches emerge from synaptic sites confirms those of others (Alsina et al., 2001; Javaherian and Cline, 2005). However, we extend these observations by showing that nascent branch initiation at synaptic sites is developmentally and locally regulated at the level of individual synapses. Thus, one mechanism by which synapses guide arbor growth is by regulating the formation of new axonal branches. New synapses promote exploratory behavior and growth, whereas at more mature synapses exploratory behavior is inhibited.

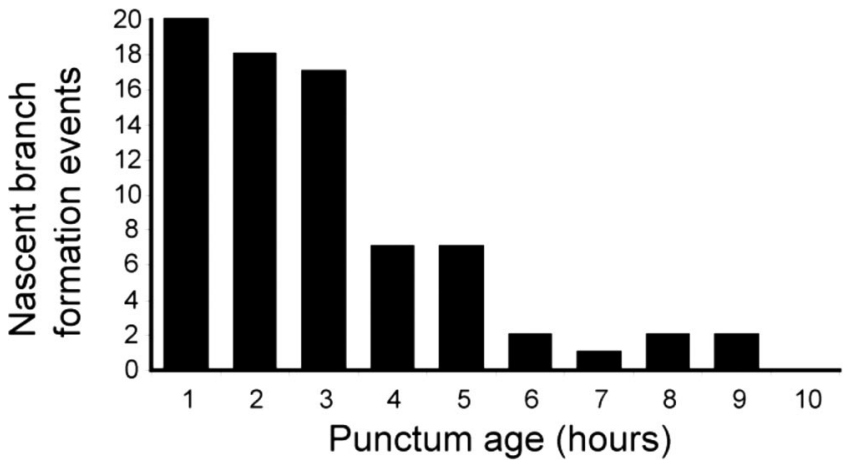

Figure 4. Exploratory behavior occurs preferentially at new Syp:GFP puncta. A bar graph showing the number of nascent branch initiation events occurring from 16 sites marked by a Syp:GFP punctum per hour of imaging period is shown. During the first hour of imaging, 20 new nascent branches emerged from the 16 sites marked by a Syp:GFP punctum, but by $6 \mathrm{~h}$ only two new nascent branches were seen to emerge from the same 16 Syp:GFP puncta. Data were obtained from four Syp:GFP-DSR-expressing cells imaged in four separate animals at $10 \mathrm{~min}$ intervals.

\section{Sites marked by Syp:GFP stabilize nascent axonal branches}

In a previous study, we showed that synapse formation stabilizes tectal cell dendritic filopodia (Niell et al., 2004). Our time-lapse analysis of RGC axonal growth demonstrates a conspicuous absence of nascent branches that persist without bearing Syp:GFP puncta. Furthermore, we also observe new nascent branches retracting to sites of Syp:GFP accumulation (Fig. $5 B$ ). These observations are consistent with the idea that sites marked by a cluster of Syp:GFP also stabilize nascent axonal branches. However, a second and equally valid interpretation of these data are that nascent branches are stabilized by a mechanism that is independent of Syp:GFP accumulation, and Syp:GFP puncta form only on previously stabilized branches. To help identify the more likely of these two alternatives, we performed four types of analysis. All quantification was performed on Syp:GFP-DSR-expressing cells imaged at $10 \mathrm{~min}$ intervals over periods of $10-15 \mathrm{~h}$.

First, we identified all newly extended nascent branches on which a single Syp:GFP punctum formed. We then measured the length of these processes as a function of time. Normalizing branch length to the position of Syp:GFP punctum formation revealed that most nascent branches extend beyond the position of Syp:GFP accumulation, but then invariably retract back to the position of the newly formed punctum. A repeated-measures ANOVA shows that nascent axon branch length differed significantly across the measurement time points $(F=10.06$; lowerbound adjusted $p=0.025$; Greenhouse-Geisser adjusted $p=$ $0.001)$. Tests for within-subject contrasts were significant for linear and quadratic trends over time. Puncta generally appear within $30 \mathrm{~min}$ of process extension and gradually increase in intensity (quantified in Fig. 5A and illustrated in Fig. 5B). The fact that processes do not stabilize at an arbitrary length suggests that the site marked by a Syp:GFP punctum plays an essential stabilizing role and implies that new axonal branches are not stabilized by a mechanism that is independent of Syp:GFP punctum formation.

Second, if the formation of a stable accumulation of Syp:GFP is required for branch stabilization, then one would predict that, in relatively mature (and hence stable) axonal arbors, every stable branch must bear a Syp:GFP punctum at its tip. To test this, we performed time-lapse imaging of a Syp:GFP-DSR-expressing cell between 9 and $10 \mathrm{dpf}$, which is when the majority of Syp:GFP puncta are stable. A series of still images taken from this time- 
lapse demonstrates, first, that most branches and puncta are indeed stable and, second, that all stable branches have a Syp:GFP cluster at their tips (Fig. 5C). This suggests that branches do not stabilize without bearing Syp:GFP puncta and that branch tips stabilize at sites marked by a stable accumulation of Syp:GFP.

Third, Figure $6 A$ shows the number of puncta on all nascent branches that were observed to appear in a time-lapse episode versus the lifetime of that branch. There is a large population of short-lived nascent branches that do not bear puncta, but with a single exception, no branch persists for $>1 \mathrm{~h}$ without bearing a Syp:GFP punctum. The single nascent branch that persisted for $>1 \mathrm{~h}$ without bearing a punctum was ultimately eliminated after $110 \mathrm{~min}$. (Averages over the lifetime of a nascent branch can be $<1$ because Syp:GFP puncta can take up to $40 \mathrm{~min}$ to appear.) These results are further characterized in a histogram comparing the number of puncta appearing on all nascent branches lasting $>1$ and $<1$ h (Fig. 6B). This shows that virtually all nascent branches that persist for $>1$ $\mathrm{h}$ bear at least one punctum for the majority of their lifetime and that $90 \%$ of branches with a lifetime of $<1 \mathrm{~h}$ do not bear puncta. These results demonstrate a clear correlation between nascent branch stability and Syp:GFP punctum formation. Furthermore, they suggest that sites marked by Syp:GFP puncta are involved in the process of nascent branch stabilization, because if axon branch stabilization were independent of Syp:GFP punctum formation, one would expect to observe some stable branches that by chance do not bear any puncta.

Finally, imaging of Syp:GFP-DSRexpressing cells reveals that not all branches bearing Syp:GFP puncta are stable indefinitely. This is illustrated by an image series taken from a time-lapse movie during which a fairly established branch bearing Syp:GFP puncta was observed to retract (Fig. 7A; supplemental video 4 , available at www.jneurosci.org as supplemental material). Before retraction of the branch, the terminal Syp:GFP punctum undergoes a gradual decrease in intensity, followed by retraction of the branch back to the adjacent punctum. The process described above is repeated, leading to a gradual stepwise retraction of the branch. By measuring the intensity of the terminal Syp:GFP punctum and the distance between the terminal Syp:GFP punctum and next proximal accumulation of Syp:GFP, we found that the terminal punctum underwent a significant decrease
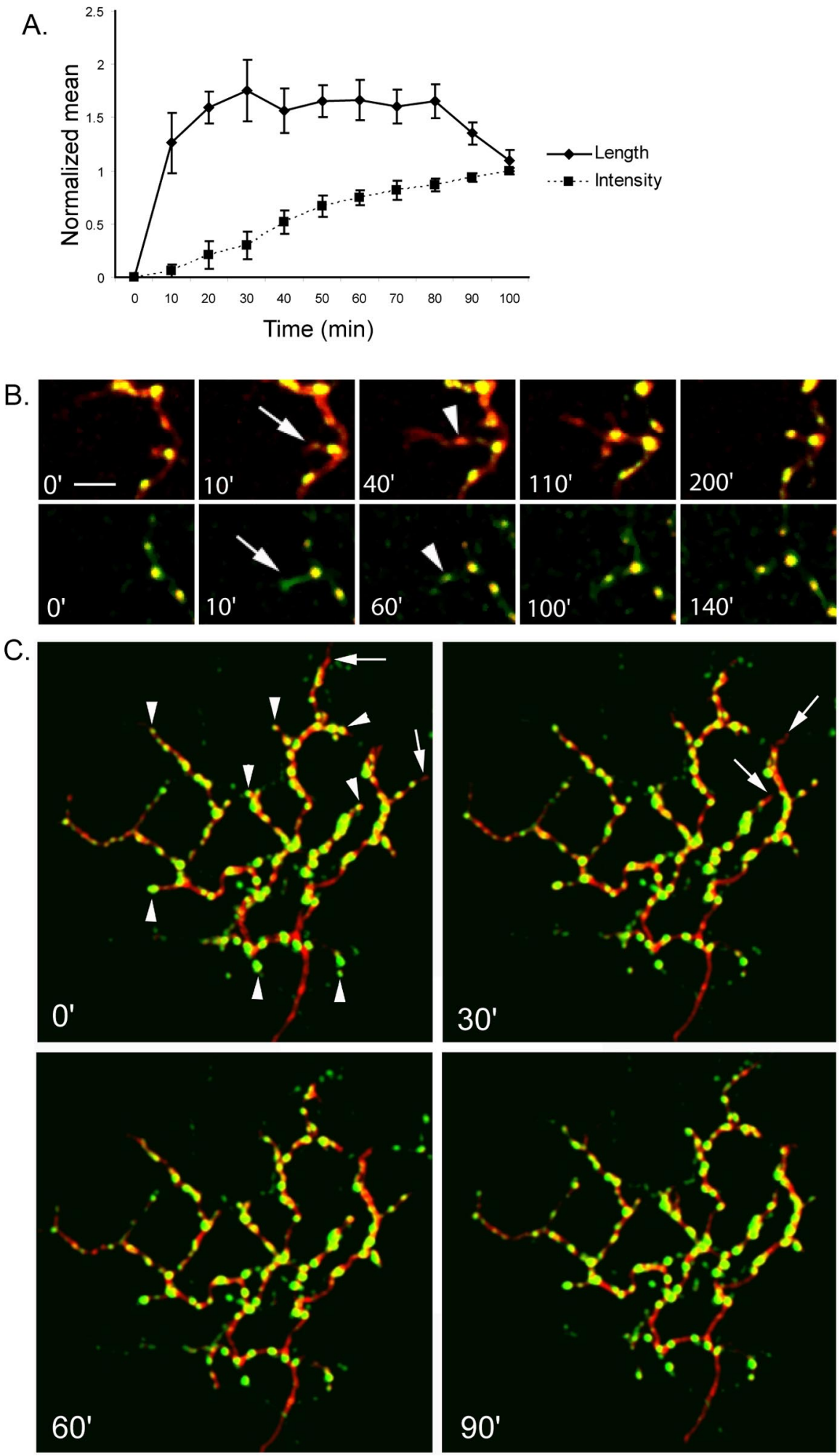

Figure 5. Nascent branches (filopodia and also those axonal processes with a growth cone at their tip) stabilize at sites of Syp:GFP accumulation. A, Quantification of all observed single-punctum formation events (average of 9 events from four Syp:GFP-DSR-expressing cells in 4 animals, imaged at 10 min intervals) shows that nascent branches retract back to the position of the stable punctum. Lengths of processes are averaged after each is normalized to a length terminating at the position of the stable punctum. Error bars indicate SEM. $\boldsymbol{B}$, Series of still images from two time-lapse sessions showing examples of nascent branches overextending and retracting back to sites of Syp:GFP accumulation. $C$, Series of still images from a time-lapse episode started at $9 \mathrm{dpf}$ demonstrates that, in mature RGC axon arbors, stable branches have a Syp:GFP cluster at their tips (arrowheads). Transient processes that did not bear a Syp:GFP are indicated by arrows. Scale bar: (in $\boldsymbol{B}$ ) $B, 5 \mu \mathrm{m} ; \boldsymbol{C}, 10 \mu \mathrm{m}$. Times are indicated in the bottom left corner of each panel. 
A.

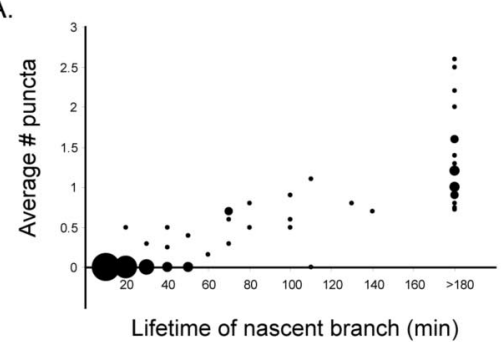

B.

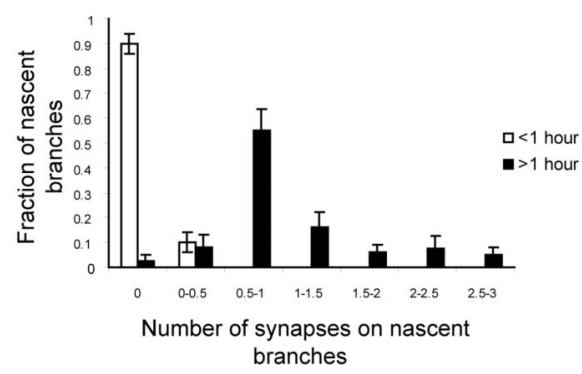

Figure 6. A, Number of puncta on newly formed nascent branches (filopodia and those processes with a growth cone at their tip) versus lifetime of that branch. Branches do not persist without bearing at least one punctum ( $n=103$ nascent branches from 4 Syp:GFP-DSR-expressing cells in 4 separate animals). Area of data point represents total number of nascent branches with a particular lifetime. $\boldsymbol{B}$, Histogram of number of puncta on all nascent branches persisting for $<1$ and $>1$ h shows that virtually no branch persists for $>1 \mathrm{~h}$ without bearing a Syp:GFP punctum and that the vast majority of processes with a lifetime of $<1 \mathrm{~h}$ bear no puncta. Error bars indicate SEM.

in fluorescence intensity well before the onset of retraction (Fig. $7 B)$. This suggests that punctum disassembly is required for branch retraction to occur. Furthermore, Figure $7 C$ demonstrates that branch retraction is a stepwise process with retraction only occurring as far back as the neighboring proximal accumulation of Syp:GFP, which stalls the retraction process until it too disassembles. If branch retraction were independent of the presence of a Syp:GFP punctum, then one would expect to see either intact Syp:GFP puncta being dragged along with the retracting branch or retracting branches stalling at arbitrary positions.

These lines of evidence together support the idea that the site marked by an accumulation of Syp:GFP plays an active role in stabilizing nascent axonal branches and that these accumulations do not merely happen to form on branches because they have been previously stabilized by a synapse independent mechanism. Such selective stabilization of nascent branches represents a second mechanism by which putative synapses marked by a cluster of Syp:GFP guide axonal arbor growth.

\section{Discussion}

To provide a detailed description of axon arbor growth and synaptogenesis, we imaged RGC axons expressing a fluorescent synaptic marker (Syp:GFP) at high temporal and spatial resolution as they arborized in the optic tectum of live zebrafish larvae. These experiments revealed that arbor growth and synaptogenesis processes are much more dynamic than could be appreciated from previous lower-time-resolution studies. That is, both nascent branches and putative nascent synapses were seen to form and disappear concurrently at much higher rates and to exhibit much shorter average lifetimes than previously realized. Correspondingly, the fraction of nascent branches and Syp:GFP puncta that were seen to stabilize using high-time-resolution imaging was found to be much smaller than expected from previous lower-time-resolution studies. Although we are by no means the first to demonstrate that axon arbor growth is a dynamic process (O’Rourke et al., 1994; Rajan et al., 1999), previous work used sample intervals of $1 \mathrm{~h}$ or greater and therefore greatly underestimated the developmental turnover kinetics that our $10 \mathrm{~min}$ sample interval now show to have major lifetime components on the scale of 20-30 min.

The time course of arbor growth and putative synapse formation that we describe here closely parallels the growth of tectal cell dendrites and appearance of postsynaptic specializations, suggesting that development of presynaptic and postsynaptic structures in the retinotectal system is highly coordinated. Further-

more, a similar degree of instability of PSD-95:GFP puncta was observed during tectal cell dendrite development (Niell et al., 2004), suggesting that the elimination of Syp:GFP puncta that we observe represents pruning of nascent synapses. This is supported by the finding that the vast majority of Syp:GFP puncta colocalize with both presynaptic and postsynaptic components in vivo (supplemental Fig. 2, available at www.jneurosci.org as supplemental material).

We find that most of these new putative nascent synapses form rapidly on newly extended nascent axonal branches, implying that the rapid morphological rearrangements of axonal arbors results in the formation of many trial synapses, only a subset of which are maintained. Thus, our imaging of arbor growth and synaptogenesis processes at high temporal resolution suggests that "trial and error" may be a much more preponderant and important element of both arbor branch and synaptic connection formation than was previously recognized.

Given the highly dynamic nature of circuit formation that we observe, an important question is, what regulates the formation and stability of nascent axonal branches? Previous studies of RGC and motor neuron axon arbor growth have shown that new axon branches emerge preferentially from synaptic sites (Alsina et al., 2001; Javaherian and Cline, 2005). Our time-lapse analysis confirms this finding, but we extend this observation by showing that nascent axonal branches occur preferentially at young synapses, and such exploratory behavior is inhibited at more mature synapses. This is consistent with the developmental regulation of axon filopodia motility by synaptic activity observed in hippocampal slice cultures (Tashiro et al., 2003). However, our analysis demonstrates that such regulation occurs at the level of individual synapses, rather than across entire cells or tissues. This mechanism may account for the fact that most growth of axonal arbors occurs preferentially from branch tips, which is where most of the newest synapses on a growing arbor are located.

It is interesting to speculate about what factors might determine the developmental shift in the ability of synapses to promote growth. At new synapses, there may be sufficient membrane components available to promote the formation of new branches, and as growth proceeds these components may become limiting. Synapse-extrinsic factors may also account for this shift. For example, as the presynaptic release machinery matures, synaptic transmission may lead to the generation of a retrograde signal from postsynaptic cells that stabilize axonal branches. The NMDA receptor-dependent stabilization of retinotectal axons implies the existence of such a retrograde signal (Rajan et al., 1999). The degree of motility of developing axons is also correlated with free extracellular space (Tashiro et al., 2003). In the developing tectal neuropil, free extracellular space would decrease over time with the addition of more axonal and dendritic branches, thereby restricting the motility of axonal branches as they matured. The demonstration that new synapses promote formation of new branches represents one mechanism by which synaptogenesis could guide growth of axonal arbors.

In addition to promoting the formation of new branches, we present several lines of evidence suggesting that putative synapses marked by a punctum of Syp:GFP also selectively stabilize nas- 
A.
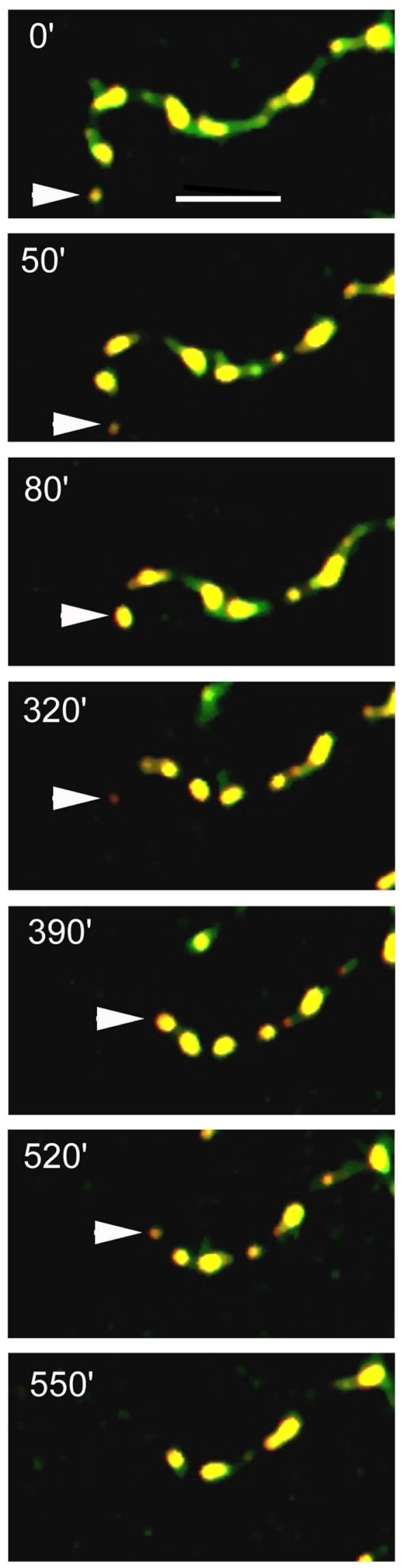

B.

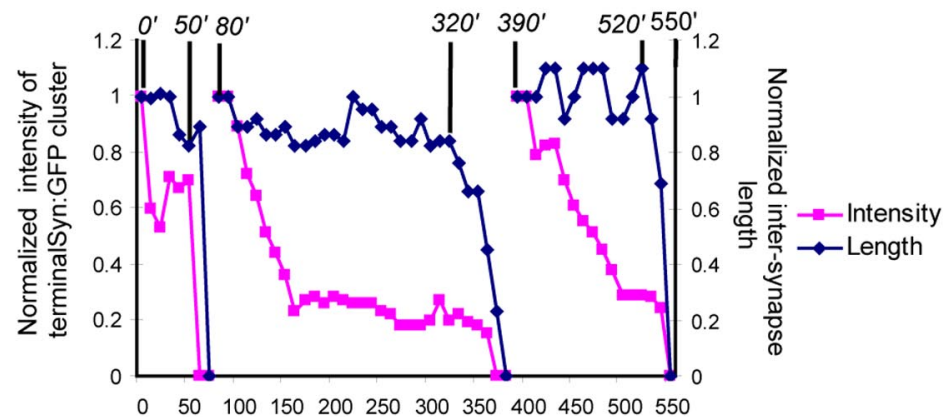

Time (minutes)

C.

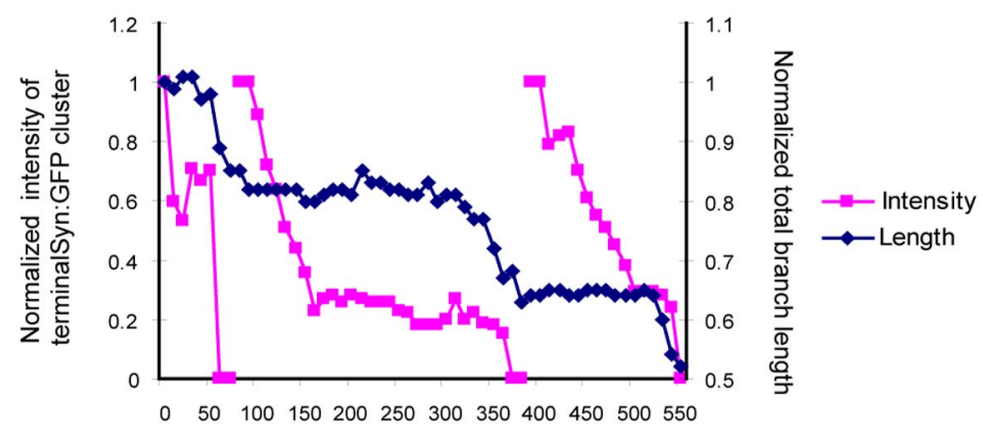

Time (minutes)
Figure 7. Imaging branch and Syp:GFP punctum elimination at $3 \mathrm{dpf} . A$, Branch elimination occurs by iterative rounds of punctum disassembly and branch retraction. A series of still images shows a decrease in intensity of a terminal punctum (arrowheads; $0-50^{\prime}$ ) precedes branch retraction to the position of the next anchoring punctum $\left(80^{\prime}\right)$. This sequence of events is then repeated $\left(320-550^{\prime}\right)$, leading to stepwise retraction of the branch. The times in minutes are indicated in the top left corner of each panel. Scale bar, $5 \mu \mathrm{m}$. B, Quantification of the retraction events depicted in $\boldsymbol{A}$ reveals that a significant degree of Syp:GFP punctum disassembly occurs before the onset of retraction. Syp:GFP punctum intensity and distance between terminal Syp:GFP cluster and next proximal cluster are normalized to the value measured at the time at which a Syp:GFP cluster becomes the most terminal. The time points illustrated in $A$ are indicated at the top of the graph. C, Comparing total branch length with the fluorescence intensity of the terminal Syp:GFP cluster demonstrates that the retraction process stalls at the position of the terminal Syp:GFP punctum and that retraction does not resume until this punctum undergoes disassembly. 
cent axonal branches. First, during the period of rapid arbor growth, new axonal growth cones and filopodia often extend beyond the point at which a Syp:GFP punctum forms, but then invariably retract back to the site of Syp:GFP accumulation, suggesting that sites marked by Syp:GFP perform a stabilizing or anchoring function. This idea is supported by that fact that, in relatively mature and hence more stable axonal arbors, all stable branches have a Syp:GFP punctum at their tip. Second, we show that nascent branches that do not form Syp:GFP puncta are rapidly eliminated and that virtually no branch persists for $>1 \mathrm{~h}$ without bearing at least one Syp:GFP punctum. These data suggest that the putative synapses that we observe are active participants in the process of branch stabilization, and argue against the notion that nascent branches are stabilized by a synapseindependent mechanism and that synapses form only on branches that are maintained for a sufficient length of time. If this scenario were true, it would be expected that, just by chance, some branches would be maintained for long periods without bearing a Syp:GFP punctum and that some branches would stabilize at arbitrary lengths.

We also observe that Syp:GFP-bearing branches are not necessarily always indefinitely stable but are sometimes eliminated within the imaging period. Such observations do not contradict the indications that putative synapses labeled with Syp:GFP stabilize nascent branches, however, because we observe that elimination of the Syp:GFP puncta precedes retraction of the corresponding branch. These observations are thus highly consistent with the hypothesized dependence of the stabilization of a given branch with the formation and persistence of a synapse on that branch.

These lines of evidence just summarized suggest that stable synapse formation is actively involved in nascent branch stabilization rather than occurring as a consequence of branch stabilization by some other means. An intriguing question then is what determines synapse stability? Adhesion between presynaptic and postsynaptic cells is almost certainly important for determining the fate of a nascent synapse. For example, the homophilic cell adhesion molecule $\mathrm{N}$-cadherin is thought to be involved in synapse maintenance (Junghans et al., 2005), and consistent with the idea that formation of a stable synapse may be important for guiding RGC arbor growth, perturbing the function of $\mathrm{N}$-cadherin, or the $\mathrm{N}$-cadherin binding partner $\beta$-catenin, disrupts laminar targeting and arborization of RGC axons within the tectum (Inoue and Sanes, 1997; Elul et al., 2003). Numerous experiments also suggest that synaptic transmission and coincident activity are important for local refinement of the retinotectal projection (Ruthazer and Cline, 2004). Effects on synapse stability could therefore mediate the effects of sensory activity on axonal arbor elaboration.

In a previous imaging study, we found that selective stabilization of filopodia by synapses is the fundamental mode of tectal cell dendrite growth (Niell et al., 2004). These data strongly support a model in which dendritic and axonal arbor growth, and synaptogenesis are linked. Iterative rounds of branch extension from existing synapses and selective stabilization of branches by formation of new synapses would bias growth of arbors in such a way as to favor additional synaptogenic contact between appropriately matched axonal and dendritic arbors. In regions devoid of appropriate synaptic partners, branches would not stabilize and net growth in these regions would not occur. Such a mechanism could provide a means for self-organization of arbor form and synaptic connections. Figure 8 depicts a model of how synaptogenesis guides growth of axonal arbors by the two distinct

\section{Guidance mechanism 1: New synapses promote axon branch formation}

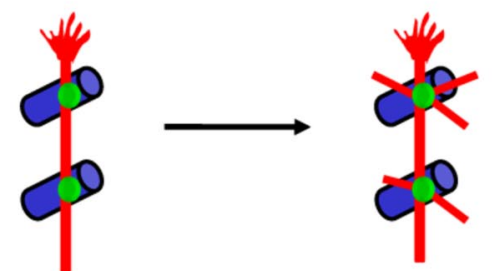

Growth cones and
filopodia establish
synaptic contacts

New synapses promote exploratory behavior and new branch formation

\section{Guidance mechanism 2: Synapses selectively stabilize new branches}

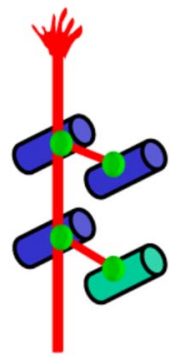

Trial contacts form

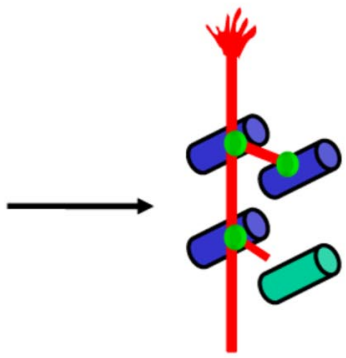

Appropriate contacts stabilize. Incorrect contacts are eliminated

\section{Iterations of mechanisms 1 and 2 lead to growth and branching.

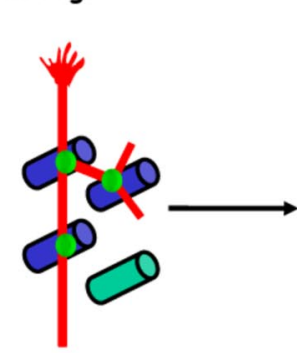 \\ Newly stabilized synapse promotes formation of new branches

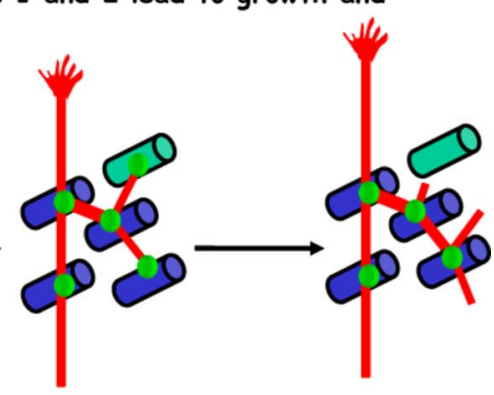 \\ Further trial contacts are made, leading to growth and branching}

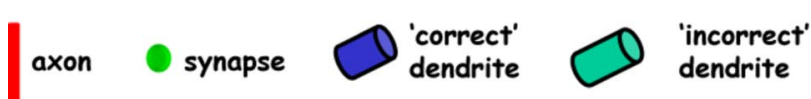

Figure 8. Synaptotropic model of RGC axon arbor growth. Synapses guide growth by promoting formation of new axon branches. New axon branches probe the environment for additional synaptic contacts. Synapses also guide growth by selectively stabilizing those branches that encounter correct partners, whereas those that establish inappropriate contacts are retracted.

mechanisms that we describe. This model is a candidate cellular mechanism underlying the guidance of axon arbor growth by synapses that was proposed over two decades ago as the "synaptotropic hypothesis" based on ultrastructural analysis of fixed tissue (Vaughn and Sims, 1978; Vaughn, 1989).

In summary, we have shown that the process of synapse formation and axon arbor growth are closely coupled. Synaptogen- 
esis guides growth of axonal arbors by (1) promoting formation of new nascent branches and (2) selectively stabilizing them. Thus, factors that regulate the formation and stability of synapses may profoundly affect axon arbor growth. By shaping axonal arbor morphology through formation of appropriate synaptic connections, a synaptotropic mode of growth may be of key importance in shaping the development of functional neural circuitry.

\section{References}

Ahmari SE, Smith SJ (2002) Knowing a nascent synapse when you see it. Neuron 34:333-336.

Ahmari SE, Buchanan J, Smith SJ (2000) Assembly of presynaptic active zones from cytoplasmic transport packets. Nat Neurosci 3:445-451.

Alsina B, Vu T, Cohen-Cory S (2001) Visualizing synapse formation in arborizing optic axons in vivo: dynamics and modulation by BDNF. Nat Neurosci 4:1093-1101.

Antonova I, Arancio O, Trillat AC, Wang HG, Zablow L, Udo H, Kandel ER, Hawkins RD (2001) Rapid increase in clusters of presynaptic proteins at onset of long-lasting potentiation. Science 294:1547-1550.

Bamji SX, Shimazu K, Kimes N, Huelsken J, Birchmeier W, Lu B, Reichardt LF (2003) Role of beta-catenin in synaptic vesicle localization and presynaptic assembly. Neuron 40:719-731.

Burrill JD, Easter Jr SS (1994) Development of the retinofugal projections in the embryonic and larval zebrafish (Brachydanio rerio). J Comp Neurol 346:583-600

Cohen-Cory S, Lom B (2004) Neurotrophic regulation of retinal ganglion cell synaptic connectivity: from axons and dendrites to synapses. Int J Dev Biol 48:947-956.

Debski EA, Cline HT (2002) Activity-dependent mapping in the retinotectal projection. Curr Opin Neurobiol 12:93-99.

Easter Jr SS, Nicola GN (1996) The development of vision in the zebrafish (Danio rerio). Dev Biol 180:646-663.

Elul TM, Kimes NE, Kohwi M, Reichardt LF (2003) N- and C-terminal domains of $\beta$-catenin, respectively, are required to initiate and shape axon arbors of retinal ganglion cells in vivo. J Neurosci 23:6567-6575.

Friedman HV, Bresler T, Garner CC, Ziv NE (2000) Assembly of new individual excitatory synapses: time course and temporal order of synaptic molecule recruitment. Neuron 27:57-69.

Goda Y, Davis GW (2003) Mechanisms of synapse assembly and disassembly. Neuron 40:243-264.

Govek EE, Newey SE, Van Aelst L (2005) The role of the Rho GTPases in neuronal development. Genes Dev 19:1-49.

Hall AC, Lucas FR, Salinas PC (2000) Axonal remodeling and synaptic differentiation in the cerebellum is regulated by WNT-7a signaling. Cell 100:525-535.

Hindges R, McLaughlin T, Genoud N, Henkemeyer M, O’Leary DD (2002) EphB forward signaling controls directional branch extension and arborization required for dorsal-ventral retinotopic mapping. Neuron 35:475-487.

Inoue A, Sanes JR (1997) Lamina-specific connectivity in the brain: regulation by $\mathrm{N}$-cadherin, neurotrophins, and glycoconjugates. Science 276:1428-1431.

Javaherian A, Cline HT (2005) Coordinated motor neuron axon growth and neuromuscular synaptogenesis are promoted by CPG15 in vivo. Neuron 45:505-512.

Jontes JD, Buchanan J, Smith SJ (2000) Growth cone and dendrite dynamics in zebrafish embryos: early events in synaptogenesis imaged in vivo. Nat Neurosci 3:231-237.

Jontes JD, Emond MR, Smith SJ (2004) In vivo trafficking and targeting of $\mathrm{N}$-cadherin to nascent presynaptic terminals. J Neurosci 24:9027-9034.

Junghans D, Haas IG, Kemler R (2005) Mammalian cadherins and pro- tocadherins: about cell death, synapses and processing. Curr Opin Cell Biol 17:446-452.

Kaether C, Skehel P, Dotti CG (2000) Axonal membrane proteins are transported in distinct carriers: a two-color video microscopy study in cultured hippocampal neurons. Mol Biol Cell 11:1213-1224.

Koster RW, Fraser SE (2001) Tracing transgene expression in living zebrafish embryos. Dev Biol 233:329-346.

Li Z, Murthy VN (2001) Visualizing postendocytic traffic of synaptic vesicles at hippocampal synapses. Neuron 31:593-605.

Li Z, Sheng M (2003) Some assembly required: the development of neuronal synapses. Nat Rev Mol Cell Biol 4:833-841.

Meyer MP, Trimmer JS, Gilthorpe JD, Smith SJ (2005) Characterization of zebrafish PSD-95 gene family members. J Neurobiol 63:91-105.

Nakata T, Terada S, Hirokawa N (1998) Visualization of the dynamics of synaptic vesicle and plasma membrane proteins in living axons. J Cell Biol 140:659-674

Niell CM, Smith SJ (2005) Functional imaging reveals rapid development of visual response properties in the zebrafish tectum. Neuron 45:941-951.

Niell CM, Meyer MP, Smith SJ (2004) In vivo imaging of synapse formation on a growing dendritic arbor. Nat Neurosci 7:254-260.

Okabe S, Miwa A, Okado H (2001) Spine formation and correlated assembly of presynaptic and postsynaptic molecules. J Neurosci 21:6105-6114.

O'Rourke NA, Cline HT, Fraser SE (1994) Rapid remodeling of retinal arbors in the tectum with and without blockade of synaptic transmission. Neuron 12:921-934.

Pennuto M, Bonanomi D, Benfenati F, Valtorta F (2003) Synaptophysin I controls the targeting of VAMP2/synaptobrevin II to synaptic vesicles. Mol Biol Cell 14:4909-4919.

Rajan I, Witte S, Cline HT (1999) NMDA receptor activity stabilizes presynaptic retinotectal axons and postsynaptic optic tectal cell dendrites in vivo. J Neurobiol 38:357-368.

Ruthazer ES, Cline HT (2004) Insights into activity-dependent map formation from the retinotectal system: a middle-of-the-brain perspective. J Neurobiol 59:134-146.

Ruthazer ES, Akerman CJ, Cline HT (2003) Control of axon branch dynamics by correlated activity in vivo. Science 301:66-70.

Shapira M, Zhai RG, Dresbach T, Bresler T, Torres VI, Gundelfinger ED, Ziv NE, Garner CC (2003) Unitary assembly of presynaptic active zones from Piccolo-Bassoon transport vesicles. Neuron 38:237-252.

Stuermer CA (1988) Retinotopic organization of the developing retinotectal projection in the zebrafish embryo. J Neurosci 8:4513-4530.

Tashiro A, Dunaevsky A, Blazeski R, Mason CA, Yuste R (2003) Bidirectional regulation of hippocampal mossy fiber filopodial motility by kainate receptors: a two-step model of synaptogenesis. Neuron 38:773-784.

Valtorta F, Pennuto M, Bonanomi D, Benfenati F (2004) Synaptophysin: leading actor or walk-on role in synaptic vesicle exocytosis? BioEssays 26:445-453.

Vaughn JE (1989) Fine structure of synaptogenesis in the vertebrate central nervous system. Synapse 3:255-285.

Vaughn JE, Sims TJ (1978) Axonal growth cones and developing axonal collaterals form synaptic junctions in embryonic mouse spinal cord. J Neurocytol 7:337-363.

Westerfield M (1995) The zebrafish book, Ed 3. Eugene, OR: University of Oregon.

Yates PA, Roskies AL, McLaughlin T, O’Leary DD (2001) Topographicspecific axon branching controlled by ephrin-As is the critical event in retinotectal map development. J Neurosci 21:8548-8563.

Zhai RG, Vardinon-Friedman H, Cases-Langhoff C, Becker B, Gundelfinger ED, Ziv NE, Garner CC (2001) Assembling the presynaptic active zone: a characterization of an active one precursor vesicle. Neuron 29:131-143.

Ziv NE, Garner CC (2004) Cellular and molecular mechanisms of presynaptic assembly. Nat Rev Neurosci 5:385-399. 